\title{
LINEAR QUADRATIC GAUSSIAN DESIGN FOR ROBUST PERFORMANCE OF A HIGHLY MANEUVERABLE AIRCRAFT ${ }^{1}$
}

by

\author{
Douglas P. Looze \\ Dept. of Electrical and Computer Engrg. \\ University of Massachusetts \\ Amherst, MA 01003
}

\author{
James S. Freudenberg \\ Dept. of Electrical Engrg. and Computer Science \\ University of Michigan \\ Ann Arbor, MI
}

\begin{abstract}
This paper describes a technique for selecting the weighting functions of the LQG/LTR formal synthesis procedure to satisfy a robust performance objective. The procedure is applied to a robust performance design problem for the longitudinal dynamics of a highly maneuverable aircraft.
\end{abstract}

\section{Introduction}

A major objective of feedback system design is to achieve a nominal performance specification for a given design model of the plant, and to maintain this performance over a range of expected errors between the design model and the true plant. Although no single performance specification can encompass all the relevant engineering design objectives, a reasonable compromise can be achieved by representing the basic performance specification as a bound on the weighted power spectrum of the input disturbances and performance outputs. Similarly, the errors between the design model and the true plant can often be reasonably represented with a nonparametric (unstructured uncertainty) model. In such cases, the structured singular value [Doy82] can be used to provide an exact measure of the robust performance of any given feedback system design.

Since the structured singular value can be used to analyze the ability of a given design to achieve robust performance, it is reasonable to also use the structured singular value directly as the objective of the feedback system design problem. One such approach has been developed in [Doy83, Doy85]. The feedback system design problem is formulated as an optimization problem, with the objective of minimizing the supremum (over all frequencies) of the structured singular value. While this approach has been successfully applied to several problems [Doy84, SkM86], it is still experimental with many difficulties remaining to be resolved.

An alternative approach to achieving a small structured singular value over all frequencies was introduced in [LoF88] In this approach, the structured singular value objective is not optimized directly. Instead, recent results in the analysis of structured singular values are used to specify characteristics of the closed loop system transfer functions that result in good robust performance [Fre88a]. These characteristics are then translated into the design parameters that are used by the formal Linear Quadratic Gaussian/Loop Transfer Function Recovery (LQG/LTR) procedure: the design weighting transfer functions on the performance, disturbance and input signals. LQG/LTR is then used to synthesize a candidate design. The design is again evaluated, and modified as required until the structured singular value performance objective is achieved.

The design problem that is considered explicitly in this paper is one of tracking an exogenous reference signal (command following) in the presence of an unstructured multiplicative modeling error at the plant input. This design problem is a typical robust performance problem, and has been studied in the context of several different applications [DoS81, FLC82, DWS82, SLH81]. It illustrates the essential features of the general robust performance problem within a more specific framework, while also providing a reasonable model for many design problems. For this problem, the characteristics of closed

1 This work was supported at the University of Michigan by the Air Force Office of Sponsored Research under contract number F33615-88-C-3601. loop systems which result in a small structured singular value have been identified and quantified. These characteristics will be mapped into desired open loop transfer function characteristics. The well known relationships between LQG/LTR design weighting functions and the subsequent compensated open loop transfer function (c.f. [DoS81, StA87]) will be used to select design weights that achieve the required robust performance characteristics. This approach has been formally presented in [LoF88] for both the LQG and $\mathrm{H}_{\infty}$ formal synthesis procedures. The approach will be illustrated in this paper by the design of a control system for the longitudinal dynamics of a highly maneuverable aircraft.

The paper is organized as follows. Section 2 formulates the problem of robust command following with input uncertainty. Section 3 summarizes results from [Fre88a, LoF88] that define design criteria in the form of desired characteristics of the compensated loop transfer function. These design criteria are then used to develop a procedure for selecting the LQG/LTR weights in Section 4. The example using these weight selections is presented in Section 5.

\section{Robust Command Following with Input Uncertainty}

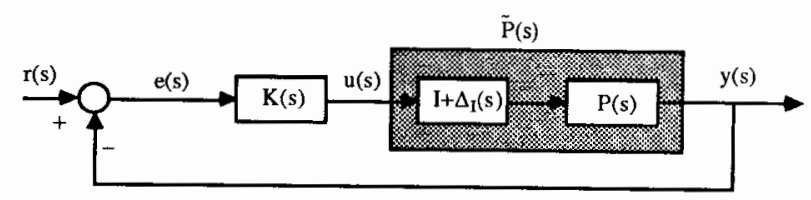

Figure 1. Robust command following system.

The system used to formulate the robust command following problem is shown in Figure 1. The control input $u(s)$, reference $\mathrm{r}(\mathrm{s})$, and measured output $\mathrm{y}(\mathrm{s})$ are all assumed to have dimension $m$. The objective of the robust command following problem is to select a compensator $\mathrm{K}(\mathrm{s})$ to ensure that the error

$$
e(s)=r(s)-y(s)
$$

is small for all reference inputs in a specified set.

The set of allowable reference inputs will be represented by a weighted Euclidean norm (denoted by $\|\cdot\|$ ). That is, $v(s)$ is an allowable exogenous input if:

$$
\left\|R_{p}^{-1}(s) r(s)\right\| \leq 1 \quad \forall s=j \omega, \omega \in \mathbb{R}
$$

where $R_{p}(s)$ is a stable, minimum-phase, proper rational transfer function. The objective of maintaining a small performance output is also represented by a weighted Euclidean norm. Thus, $\mathrm{e}(\mathrm{s})$ is an acceptable output if

$$
\left\|\mathrm{L}_{\mathrm{p}}(\mathrm{s}) \mathrm{e}(\mathrm{s})\right\|<1 \quad \forall \mathrm{s}=\mathrm{j} \omega, \omega \in \mathbb{R}
$$

where $L_{p}(s)$ is a stable, minimum-phase, proper rational transfer function.

The robust performance objective is to satisfy the nominal performance objective (2)-(3) for every allowable plant transfer function $\widetilde{P}(s)$ in Figure 1 . The set of allowable plant errors is represented by a multiplicative error model (see Figure 1), with the multiplicative uncertainty block modeled as a nonparametric (unstructured) uncertainty that is unknown except for its 
magnitude as measured by a weighted singular value norm bound:

$$
\bar{\sigma}\left[\mathrm{R}_{\mathrm{u}}^{-1}(\mathrm{~s}) \Delta_{\mathrm{I}}(\mathrm{s}) \mathrm{L}_{\mathrm{u}}^{-1}(\mathrm{~s})\right] \leq 1 \quad \forall \mathrm{s}=\mathrm{j} \omega, \omega \in \mathbb{R}
$$

where $R_{u}(s)$ and $L_{p}(s)$ are stable, minimum-phase, proper rational transfer functions.

The robust performance objective is to satisfy (3) for all external references that satisfy (2) and all multiplicative error sources that satisfy (4). This objective can be reformulated as a robust stability problem by inserting a fictitious divisive error $\Delta \mathrm{P}(\mathrm{s})$ at the plant output (c.f., [DWS82]). The fictitious performance error is also modeled as a nonparametric error source, that satisfies:

$$
\bar{\sigma}\left[\mathrm{R}_{\mathrm{p}}^{-1}(\mathrm{~s}) \Delta_{\mathrm{P}}(\mathrm{s}) \mathrm{L}_{\mathrm{p}}^{-1}(\mathrm{~s})\right] \leq 1 \quad \forall \mathrm{s}=\mathrm{j} \omega, \omega \in \mathbb{R}
$$

The closed loop system can then be modeled as shown in Figure 2. The (normalized) closed loop transfer function $\mathrm{G}(\mathrm{s})$ is given by:

$$
\underline{G}(s)=\left[\begin{array}{cc}
-L_{u}(s) T_{I}(s) R_{u}(s) & L_{u}(s) P^{-1}(s) T_{O}(s) R_{p}(s) \\
-L_{p}(s) P(s) S_{I}(s) R_{u}(s) & L_{p}(s) S_{O}(s) R_{p}(s)
\end{array}\right]
$$

where

$$
\begin{aligned}
& \mathrm{S}_{\mathrm{O}}(\mathrm{s})=[\mathrm{I}+\mathrm{P}(\mathrm{s}) \mathrm{K}(\mathrm{s})]^{-1} \\
& \mathrm{~T}_{\mathrm{O}}(\mathrm{s})=\mathrm{P}(\mathrm{s}) \mathrm{K}(\mathrm{s})[\mathrm{I}+\mathrm{P}(\mathrm{s}) \mathrm{K}(\mathrm{s})]^{-1} \\
& \mathrm{~S}_{\mathrm{I}}(\mathrm{s})=[\mathrm{I}+\mathrm{K}(\mathrm{s}) \mathrm{P}(\mathrm{s})]^{-1} \\
& \mathrm{~T}_{\mathrm{I}}(\mathrm{s})=\mathrm{K}(\mathrm{s}) \mathrm{P}(\mathrm{s})[\mathrm{I}+\mathrm{K}(\mathrm{s}) \mathrm{P}(\mathrm{s})]^{-1}
\end{aligned}
$$

and where the normalized error $\Delta(\mathrm{s})$ is given by:

$$
\Delta(\mathrm{s})=\left[\begin{array}{cc}
\mathrm{R}_{\mathrm{u}}^{-1}(\mathrm{~s}) \Delta_{\mathrm{I}}(\mathrm{s}) \mathrm{L}_{\mathrm{u}}^{-1}(\mathrm{~s}) & 0 \\
0 & \mathrm{R}_{\mathrm{p}}^{-1}(\mathrm{~s}) \Delta_{\mathrm{P}}(\mathrm{s}) \mathrm{L}_{\mathrm{p}}^{-1}(\mathrm{~s})
\end{array}\right]
$$

The system of Figure 2 is stable for all $\Delta(\mathrm{s})$ given by (7) with ${ }^{1}$

$$
\bar{\sigma}[\Delta(\mathrm{s})] \leq 1 \quad \forall \mathrm{s}=\mathrm{j} \omega, \omega \in \mathbb{R}
$$

if and only if the system of Figure 1 satisfies the performance objective (3) for all reference inputs that satisfy (2) and all model errors that satisfy (4).

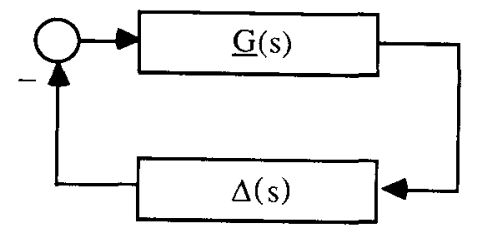

Figure 2. System with robust stability condition equivalent to the robust performance condition.

The robust stability problem with a diagonal error structure such as (8) can be evaluated by the structured singular value [Doy82]. The structured singular value $\mu$ is defined as a function of a matrix and a block diagonal perturbation structure The perturbation structure is given by an ordered set $\mathbf{k}=\left\{\mathrm{k}_{1}, \ldots, \mathrm{k}_{\mathrm{k}}\right\}$ that specifies the dimensions of the diagonal blocks ${ }^{2}$. Let the set of allowable perturbations be given as:

$D_{\mathbf{k}}=\left\{\operatorname{diag}\left[\Delta_{\mathrm{i}}: \mathrm{i}=1, \ldots, \mathrm{k}\right]: \Delta_{\mathrm{i}} \in \mathbb{C}^{\mathrm{k}_{\mathrm{i}} \times \mathrm{k}_{\mathrm{i}}}\right\}$

The structured singular value of a matrix with respect to this structure is defined as:
The structure of the perturbation (8) to the system in Figure 2 is defined by $k=\{m, m\}$. The structured singular value can be applied to the normalized closed loop transfer function [DWS82] to provide a necessary and sufficient condition for the robust stability of the system in Figure 2, and consequently for the robust performance of the system in Figure 1. Thus, a given compensator $K(\mathrm{~s})$ satisfies the robust performance condition for all plant model errors satisfying (4) and all reference signals satisfying (2) if and only if:

$$
\mu[\underline{\mathrm{G}}(\mathrm{s})]<1 \quad \forall \mathrm{s}=\mathrm{j} \omega, \omega \in \mathbb{R}
$$

\section{Analysis of a Simplified Robust Performance} Problem

The objective of this section is to identify design problem characteristics that affect the structured singular value, and quantify the relationship between the structured singular value and those characteristics. A number of tradeoffs imposed by the algebraic loop constraints and the stability [FrL88, SLH81, Doy79] that apply to the general control system design problem will apply here. Also, ill-conditioned plant transfer functions can cause problems when simultaneous input and output uncertainties are present in the plant model [Ste84, FrL86b, NeM87, SkM86]. This section will summarize recent results [Fre88a, LoF88] that bound the structured singular value in terms of the conditioning of the plant. The results will be presented for two-input, two-output systems (i.e., $m=2$ ) with uniform performance and uncertainty weighting functions. More general statements of these results can be found in [Fre88a].

Assume that the plant transfer function $\mathrm{P}(\mathrm{s})$ has the singular value decomposition ${ }^{1}$ :

$$
\begin{aligned}
\mathrm{P}(\mathrm{s}) & =\mathrm{W}(\mathrm{s}) \mathrm{T}(\mathrm{s}) \mathrm{Z}^{\mathrm{H}}(\mathrm{s}) \\
& =\sum_{\mathrm{i}=1}^{2} w_{\mathrm{i}} \mathrm{z}_{\mathrm{i}}^{\mathrm{H}} \tau_{\mathrm{i}}
\end{aligned}
$$

where the matrices $W=\left[w_{1} w_{2}\right]$ and $Z=\left[z_{1} z_{2}\right]$ are unitary and $\mathrm{T}=\operatorname{diag}\left[\tau_{1}, \tau_{2}\right]$ with $\tau_{1} \geq \tau_{2}$. The condition number of the plant $P(s)$ at a frequency $s$ is defined as:

$$
\kappa_{\mathrm{p}}(\mathrm{s})=\frac{\tau_{1}(\mathrm{~s})}{\tau_{2}(\mathrm{~s})}
$$

For a given compensator $\mathrm{K}(\mathrm{s})$, define the loop transfer function at the output $\mathrm{L}_{\mathrm{O}}(\mathrm{s})$ as:

$\mathrm{L}_{\mathrm{O}}(\mathrm{s})=\mathrm{P}(\mathrm{s}) \mathrm{K}(\mathrm{s})$

and the loop transfer function at the input as:

$\mathrm{L}_{\mathrm{I}}(\mathrm{s})=\mathrm{K}(\mathrm{s}) \mathrm{P}(\mathrm{s})$

The performance objectives and plant uncertainty are assumed to be uniform in the signal directions:

$$
\begin{array}{ll}
\mathrm{L}_{\mathrm{u}}(\mathrm{s})=\pi_{\mathrm{u}}(\mathrm{s}) \mathrm{I} & \mathrm{R}_{\mathrm{u}}(\mathrm{s})=\mathrm{I} \\
\mathrm{L}_{\mathrm{p}}(\mathrm{s})=\pi_{\mathrm{p}}(\mathrm{s}) \mathrm{I} & \mathrm{R}_{\mathrm{p}}(\mathrm{s})=\mathrm{I}
\end{array}
$$

where $w_{\mathrm{u}}(\mathrm{s})$ and $\mathrm{w}_{\mathrm{p}}(\mathrm{s})$ are rational scalar functions of $\mathrm{s}$.

The structured singular value can be approximated in terms of the closed loop sensitivity and transfer functions using the results in [Fre88a]. A key quantity in this approximation is the weighted condition number $\underline{\mathrm{K}}$, which is obtained by combining the uniform weighting functions with the plant condition number:

$$
\underline{\kappa} \mathrm{P}(\mathrm{s})=\left|\pi_{\mathrm{u}}(\mathrm{s}) \pi_{\mathrm{p}}(\mathrm{s})\right| \kappa_{\mathrm{P}}(\mathrm{s})
$$

$$
\mu[\mathrm{M}]= \begin{cases}0 & \text { if } \operatorname{det}(\mathrm{I}+\mathrm{M} \Delta) \neq 0 \forall \Delta \in D_{\mathbf{k}} \\ {\left[\min _{\Delta \in D_{\mathbf{k}}}\{\bar{\sigma}[\Delta]: \operatorname{det}(\mathrm{I}+\mathrm{M} \Delta)=0\}\right]^{-1}} & \text { otherwise }\end{cases}
$$

1 To simplify notation and clarify the presentation, the dependence of any quantity on the complex frequency variable $s$ will be omitted when this dependency is not crucial to the discussion.

\footnotetext{
1 i.e., for all $\Delta_{\mathrm{I}}(\mathrm{s})$ satisfying (4) and $\Delta \mathrm{P}(\mathrm{s})$ satisfying (5)

2 We assume for simplicity that there are no repreated blocks. Sce [Doy82] for the more general definition.
} 
Then, the results of [Fre88a] state that if the structured singular value is less than unity (and consequently the robust performance objective is achieved) then:

$$
\left\|\mathrm{S}_{\mathrm{O}} \mathrm{w}_{1}\right\|\left\|\mathrm{w}_{2}^{\mathrm{H}} \mathrm{T}_{\mathrm{O}}\right\|<\underline{\kappa}_{\mathrm{P}}^{-1}
$$

or

$$
\left\|T_{I} z_{2}\right\|\left\|z_{1}^{H} S_{I}\right\|<\underline{\kappa}_{P}^{-1}
$$

Thus, to make the structured singular value small, it is necessary to have the left hand sides of (20)-(21) small. When the singular values of the loop transfer functions $\left(\mathrm{LO}_{\mathrm{O}}\right.$ and $\left.\mathrm{L}_{\mathrm{I}}\right)$ are either all large or all small, (20)-(21) will be satisfied. If one of the singular values of the loop transfer functions, say $\mathrm{L}_{\mathrm{I}}(\mathrm{s})$, is near unity, then there will be one singular value of both $S_{I}$ and $\mathrm{T}_{\mathrm{I}}$ of order one. To make the left hand side of (20) small, either So must be small in the right subspace $\mathrm{w}_{1}$ or $\mathrm{T}_{\mathrm{O}}$ must be small in the left subspace w2. As shown in [FrL86a], such conditions imply that a singular value of the loop transfer function Lo must either be large with left singular subspace $w_{1}$, or small with left singular subspace w2. Condition (20) imposes analogous requirements on the singular values and right singular subspaces of $\mathrm{L}_{\mathrm{I}}$. Thus, the compensator $\mathrm{K}(\mathrm{s})$ must not significantly modify the singular subspace structure of the plant in the crossover region. To achieve this, the singular value decomposition of the loop transfer functions near the crossover region must approximately be:

$$
\begin{aligned}
& \mathrm{LO}_{\mathrm{O}}(\mathrm{s}) \approx \mathrm{W}(\mathrm{s}) \Sigma(\mathrm{s}) \mathrm{W}(\mathrm{s})^{\mathrm{H}} \\
& \mathrm{L}_{\mathrm{I}}(\mathrm{s}) \approx \mathrm{Z}(\mathrm{s}) \Sigma(\mathrm{s}) \mathrm{Z}(\mathrm{s})^{\mathrm{H}}
\end{aligned}
$$

where

$$
\Sigma(\mathrm{s})=\left[\begin{array}{cc}
l_{1}(\mathrm{~s}) & 0 \\
0 & l_{2}(\mathrm{~s})
\end{array}\right]
$$

References [FrL86a,Fre88b] quantify and analyze the reduction in nominal performance that will result from a misalignment of the left and right singular subspaces.

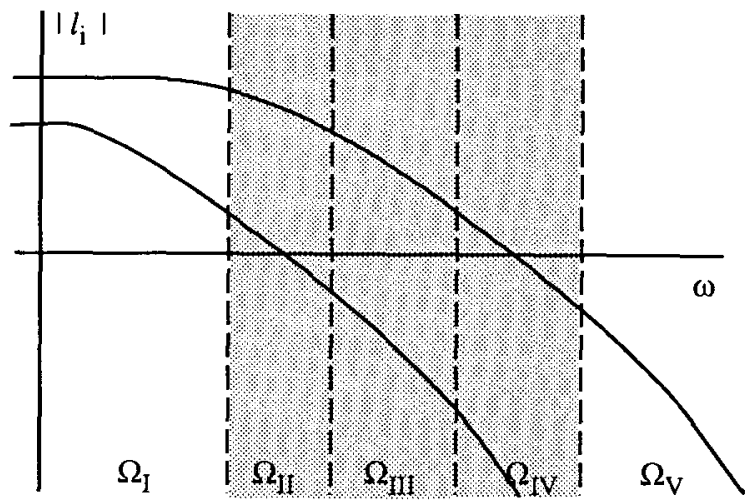

Figure 3. Typical loop shapes for a 2-input, 2-output system.

The consequences of this discussion are illustrated in Figure 3 for the $2 \times 2$ system with uniform weightings. The frequency range is divided into five regions: region $\Omega_{\text {I }}$ in which both singular values of the loop transfer function are large; region $\Omega_{\text {II }}$ in which one of the singular values is large, but the other is near unity; region $\Omega_{\mathrm{III}}$ in which one singular value is large, and the other singular value is small; region $\Omega_{\mathrm{IV}}$ in which one of the singular values is small, and the other is near unity; and region $\Omega_{\mathrm{V}}$ in which both singular values are small. The conditions for robust performance in regions $\Omega_{\mathrm{I}}$ and $\Omega_{\mathrm{V}}$ can be found by the approximation $\mathrm{T}_{\mathrm{I}} \approx \mathrm{I}$ in region $\Omega_{\mathrm{I}}$ and $\mathrm{S}_{\mathrm{I}} \approx \mathrm{I}$ in region $\Omega_{\mathrm{V}}$. These conditions can be stated as:

$$
\begin{array}{ll}
\bar{\sigma}\left[L_{I}(s)\right]>\underline{\kappa} P(s) & \forall s=j \omega, \omega \in \Omega_{I} \\
g\left[L_{I}(s)\right]<\underline{\kappa}_{P}^{-1}(s) & \forall s=j \omega, \omega \in \Omega_{V}
\end{array}
$$

In addition, the singular value conditions conditions for nominal performance and robust stability must be satisfied in these regions. Using properties of the LQ regulator, the loop transfer function at the input should satisfy

$$
\begin{array}{ll}
\underline{\sigma}\left[\mathrm{L}_{\mathrm{I}}(\mathrm{s})\right]>\pi_{\mathrm{p}}(\mathrm{s}) & \forall \mathrm{s}=\mathrm{j} \omega, \omega \in \Omega_{\mathrm{I}} \\
\bar{\sigma}\left[\mathrm{L}_{\mathrm{I}}(\mathrm{s})\right]<\pi_{\mathrm{u}}^{-1}(\mathrm{~s}) & \forall \mathrm{s}=\mathrm{j} \omega, \omega \in \Omega_{\mathrm{V}}
\end{array}
$$

In regions $\Omega_{\mathrm{II}}, \Omega_{\mathrm{III}}$, and $\Omega_{\mathrm{IV}}$ the loop transfer function must satisfy (22). Thus, the singular values of the input sensitivity and transfer functions are approximately:

$$
\begin{array}{lll}
\mathrm{s}_{\mathrm{i}}=\frac{1}{1+l_{\mathrm{i}}} & \mathrm{i}=1,2 \\
\mathrm{t}_{\mathrm{i}}=\frac{l_{\mathrm{i}}}{1+l_{\mathrm{i}}} & \mathrm{i}=1,2
\end{array}
$$

Condition (20) becomes:

$$
\left|s_{1} t_{2}\right|<k_{P}^{-1}
$$

In region $\Omega_{\mathrm{II}}, t_{2}$ is near unity and $s_{1}^{-1}$ is approximately $l_{1}$. Thus,

$$
\bar{\sigma}\left[L_{\mathrm{I}}(\mathrm{s})\right]>\underline{\kappa_{P}}(\mathrm{~s}) \quad \forall \mathrm{s}=\mathrm{j} \omega, \omega \in \Omega_{\mathrm{II}}
$$

In region $\Omega_{\mathrm{III}}, \mathrm{t}_{2}$ is approximately $l_{2}$ and $\mathrm{s}_{1}^{-1}$ is approximately $l_{1}$. Thus,

$$
\bar{\sigma}\left[\mathrm{L}_{\mathrm{I}}(\mathrm{s})\right]>\underline{\mathrm{K}} \mathrm{P}(\mathrm{s}) \Phi\left[\mathrm{L}_{\mathrm{I}}(\mathrm{s})\right] \quad \forall \mathrm{s}=\mathrm{j} \omega, \omega \in \Omega_{\mathrm{III}}
$$

In region $\Omega_{\mathrm{IV}}, \mathrm{t}_{2}$ is approximately $l_{2}$ and $\mathrm{s}_{1}^{-1}$ is approximately unity. Thus,

$$
\underline{\sigma}\left[\mathrm{L}_{\mathrm{I}}(\mathrm{s})\right]<\underline{\underline{K}}_{\mathrm{P}}^{-1}(\mathrm{~s}) \quad \forall \mathrm{s}=\mathrm{j} \omega, \omega \in \Omega_{\mathrm{IV}}
$$

To summarize, the loop transfer function of the plant at the input must satisfy the singular subspace alignment condition (23) in the crossover range $\Omega_{\mathrm{II}} \cup \Omega_{\mathrm{III}} \cup \Omega_{\mathrm{IV}}$. The singular values of the loop transfer function must satisfy (25)-(28) and (32)-(34). Conditions (32)-(34) imply that the loop singular values must be separated by at least the weighted condition number $\mathrm{kp}$ over the interior of the crossover range, while the bounds in (25)-(28) indicate that a separation proportional to the plant condition number is desirable. Analogous conditions can be stated for the loop transfer function at the output.

While conditions (32)-(34) impose restrictions on the separation in magnitude of the singular values of the loop transfer function, they do not directly affect the width of the crossover region. That is, by allowing the singular values to decrease arbitrarily rapidly, the crossover region could be made arbitrarily small while maintaining the required separation between the singular values. However, as in scalar systems, the rate of decrease of the loop singular values is limited. To show this, we relax the convention that the singular values of the loop transfer functions be real, and assign an angle to each of the singular values using the techniques developed in [FrL84, FrL88]. Thus, each $l_{\mathrm{i}}(\mathrm{s})$ will have the same magnitude as before and the singular vectors of the loop span the same subspaces as in the standard singular value decomposition. Now, however, the functions $l_{i}(s)$ are complex valued.

We can quantify the required spread between the crossover frequencies and identify the features of the problem that contribute to this limitation by examining the relationships between the open loop transfer functions $l_{1}(\mathrm{~s})$ and $l_{2}(\mathrm{~s})$ and the value of the product $\left|s_{1} t_{2}\right|$. Since $l_{2}(s)$ crosses over before $l_{1}(s)$, we have:

$$
\left|l_{1}\right| \gg 1,\left|l_{2}\right| \approx 1 \Rightarrow\left|s_{1} t_{1}\right|=\frac{1}{\left|l_{1}\right|} \frac{1}{\left|2 \sin \left(\frac{\pi+\angle l_{2}}{2}\right)\right|}
$$

Combining (32) and (35) shows that the lower bound on the structured singular value will be less than one near the second loop crossover if 


$$
\left|l_{1}\right|>\frac{k p}{\left|2 \sin \left(\frac{\pi+\angle l_{2}}{2}\right)\right|}
$$

Similarly, the lower bound (34) will be satisfied near the crossover of the first loop if

$$
k_{2} \mid>\frac{\left|2 \sin \left(\frac{\pi+\angle l_{1}}{2}\right)\right|}{\kappa \mathrm{sP}}
$$

The bounds (36)-(37) describe relationships between the magnitudes of the open loop transfer functions and the generalized phases of the open loop transfer functions that provide more information than the corresponding bounds (32) and (34). Recall that if the loop transfer functions $l_{i}(s)$ are analytic in $\mathbb{C}^{+}$, the Bode gain-phase relation $[\operatorname{Bod} 45]$ could be used to show the existence of a tradeoff between the rate of gain decrease and the phase margin in each loop. Indeed, the need to satisfy the bounds (36)-(37) may require that each loop roll off more rapidly than if only the bounds (27)-(28) that represent the nominal objectives were present. For the general multivariable case in which the transfer functions $l_{\mathrm{i}}(\mathrm{s})$ may not be analytic, the generalization of the Bode relation [FrL85a, FrL87] plays a similar role. In particular, if either loop gain is decreased too rapidly near crossover then the phase angle of that loop at crossover will be large. The bound that utilizes this phase angle then imposes a more severe requirement on the other loop. This in turn requires a greater decrease in the other loop, which decreases its phase margin.

The structured singular value objective requires that (27)(28), (33) and (36)- (37) be satisfied by the loop transfer function. Thus, the objective of the robust performance design problem is to select a compensator such that these conditions are fulfilled.

\section{Selection of LQG/LTR Weighting Functions}

The objective of this paper is to use the LQG/LTR synthesis method to design a compensator that satisfies structured singular value objectives such as (12). The preceding section presented design conditions that describe the characteristics of the compensator that are needed to achieve the desired robust performance for ill-conditioned systems for a simplified problem with uniform objectives and uncertainties. These conditions are stated in terms of the compensated open loop transfer function at the plant input. This section will present a procedure for selecting the LQG/LTR weighting functions for this simplified problem.

The LQG/LTR procedure (for input recovery) can be viewed in the frequency domain in terms of the system shown in Figure 4 (see [StA 87]). The exogenous signal $\xi(\mathrm{s})$ (the process noise) is assumed to be a Gaussian white noise signal. The formal objective of the LQG/LTR procedure is to minimize the variance of the performance signals $v(s)$ and $z(s)$. The signals $v(s)$ and $\mathrm{z}(\mathrm{s})$ are used to appropriately penalize the input and output and output signals, respectively. The LQG/LTR objective is expressed mathematically as:

$$
\min _{K(s)}\left\{\int_{-\infty}^{\infty} G_{s}^{T}(-j \omega) G_{s}(j \omega) d \omega\right\}
$$

where $G_{s}(s)$ is the transfer function from the process noise $\xi(s)$ to the penalized signals $v(s)$ and $z(s)$ :

$$
G_{s}(s)=\left[\begin{array}{c}
-W_{u}(s) T_{I}(s) W_{\xi}(s) \\
W_{p}(s) P(s) S_{I}(s) W_{\xi}(s)
\end{array}\right]
$$

The transfer functions $W_{u}(s)$ and $W_{\xi}(s)$, together with the recovery procedure, represent the design parameters of the LQG/LTR design process.

Note that the transfer function $G_{s}(s)$ is closely related to the transfer function $\mathrm{G}(\mathrm{s})$ that determines the structured singular value measure of robust performance (6). By selecting the LQG/LTR design parameters as:

$$
\begin{aligned}
& \mathrm{W}_{\mathrm{p}}(\mathrm{s})=\pi_{\mathrm{p}}(\mathrm{s}) \mathrm{W}_{\mu}(\mathrm{s}) \\
& \mathrm{W}_{\xi}(\mathrm{s})=\mathrm{I} \\
& \mathrm{W}_{\mathrm{u}}(\mathrm{s})=\pi_{\mathrm{u}}(\mathrm{s}) \mathrm{I}
\end{aligned}
$$

the transfer function $G_{S}(s)$ is the same as the first block of columns of $\mathrm{G}(\mathrm{s})$. Thus, making $\mathrm{G}_{\mathrm{s}}(\mathrm{s})$ small with the selections (40)-(42) will also tend to make $\underline{G}(\mathrm{~s})$ small.

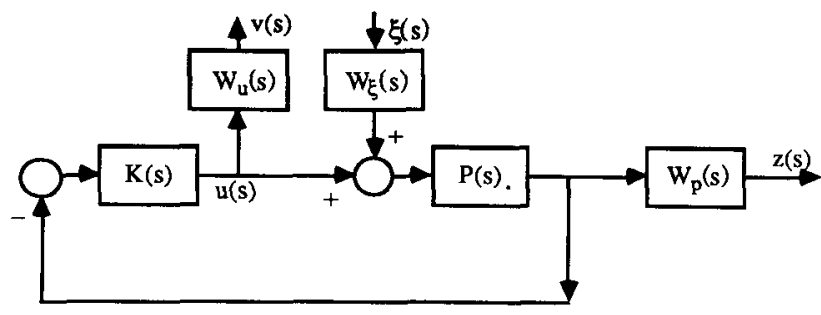

Figure 4. The mixed sensitivity problem.

Our approach will utilize this observation to initialize the design procedure. That is, we will the formal synthesis weights to be as near as possible to the weighting functions that describe the nominal performance and robustness objectives, and to adjust them only to enforce the singular value separation conditions (25)-(34). Thus, our initial selection of the design parameters is given by (40)-(42).

Further motivation for these selections is obtained from properties of the LQG/LTR design. Since the complete weighting function that multiplies $S_{I}(s)$ is $\pi_{\mathrm{D}}(\mathrm{s}) \mathrm{P}(\mathrm{s})$ (see (38) and (40)), the sensitivity function must satisfy alignment conditions such as (21) and the ones analyzed in [FrL86a]. Using the arguments presented in [Fre86a], it can be shown that (22)-(23) must be satisfied. Thus, the only conditions remaining to be satisfied are (25)-(34).

The latter conditions can be enforced by iteratively selecting the weighting function $W_{\mu}(s)$ and adjusting the bandwidth of the recovery process. The individual singular values of the loop transfer function can be manipulated in a frequency range $\Omega_{\mathrm{m}}$ in the crossover region by selecting

$$
\mathrm{W}_{\mu}(\mathrm{s})=\mathrm{Z}\left[\begin{array}{ll}
\underline{\mathrm{a}} & 0 \\
0 & \underline{\mathrm{b}}
\end{array}\right] \mathrm{Z}^{\mathrm{H}} \quad \mathrm{s} \in \Omega_{\mathrm{m}}
$$

Since the transfer function $T_{I}(s)$ has approximately the same singular subspaces as the plant right singular subspaces, the value a will change the largest singular value of the loop transfer function by approximately the factor $\mathbf{a}$. Similarly, the value $\underline{b}$ will change the smallest singular value of the loop transfer function by approximately the factor $\underline{b}$. Thus, the parameters $\underline{a}$ and $b$ can be used to spread the singular values apart, or to squeeze them together while still satisfying the nominal objectives (27)-(28). The low frequency separation condition implied by (25) and (27) will automatically be satisfied by this choice of weights. To allow the high frequency plant condition number to enforce the high frequency separation condition implied by (26) and (28), the recovery bandwidth will be selected so that the loop is recovered to just beyond the last loop crossover.

There is one additional consideration when using the LQG/LTR formal synthesis technique. As noted in [ZaF83], the LQG objectives penalize high frequencies more than are reflected in the robust performance objective stated in Section 2. This results in LQG/LTR designs that have one additional pole rolloff at high frequencies than is necessary (see[BSM87]). Thus, the LQG/LTR weights will be adjusted to add an additional high frequency lead and remove the extra high frequency rolloff. This is accomplished by adding a lag filter $\pi l(s)$ to the weighting function $\mathrm{W}_{\mathrm{u}}(\mathrm{s})$ :

$$
\mathrm{W}_{\mathrm{u}}(\mathrm{s})=\pi_{\mathrm{u}}(\mathrm{s}) \pi_{l}(\mathrm{~s}) \mathrm{I}
$$




\section{Robust Performance for a Highly Maneuverable Aircraft}

We will consider the problem of designing a robust performance longitudinal control system for a highly maneuverable aircraft. This problem was originally considered in [Ste84]. The aircraft characteristics and linearized models are discussed in detail in [HBG79]. The aircraft possesses two sets of longitudinal control surfaces: elevators and canards. Two measurement signals, angle of attack and pitch angle, are assumed to be available. For this problem, a stable version of the aircraft model is used. The linearized model of the longitudinal dynamics at mach 0.9 , altitude $25,000 \mathrm{ft}$. is given by:

$$
\begin{aligned}
& \dot{x}(t)=A x(t)+B u(t) \\
& y(t)=C x(t)
\end{aligned}
$$

where

$$
\begin{aligned}
& A=\left[\begin{array}{cccc}
-0.0226 & -36.6 & -18.9 & -32.1 \\
0 & -1.9 & -0.983 & 0 \\
0.0123 & -11.7 & -2.63 & 0 \\
0 & 0 & 1.0 & 0
\end{array}\right] B=\left[\begin{array}{cc}
0 & 0 \\
-0.414 & 0 \\
-77.8 & 22.4 \\
0 & 0
\end{array}\right] \\
& C=\left[\begin{array}{cccc}
0 & 57.3 & 0 & 0 \\
0 & 0 & 0 & 57.3
\end{array}\right]
\end{aligned}
$$

This plant model is stable and minimum phase, with a large condition number at most frequencies. The singular values of the model are shown in Figure 5. The plant condition number (in $\mathrm{db}$ ) is observed in Figure 5 as the difference between the largest and smallest singular values. The physical source for the large condition number is the ability of the control surfaces to impart relatively large amounts of rotational energy to the aircraft (and hence a large transfer function from inputs to pitch angle), combined with an inability to transmit relatively large amounts of kinetic energy to the aircraft (and hence a smaller transfer function from inputs to vertical velocity, or angle of attack).

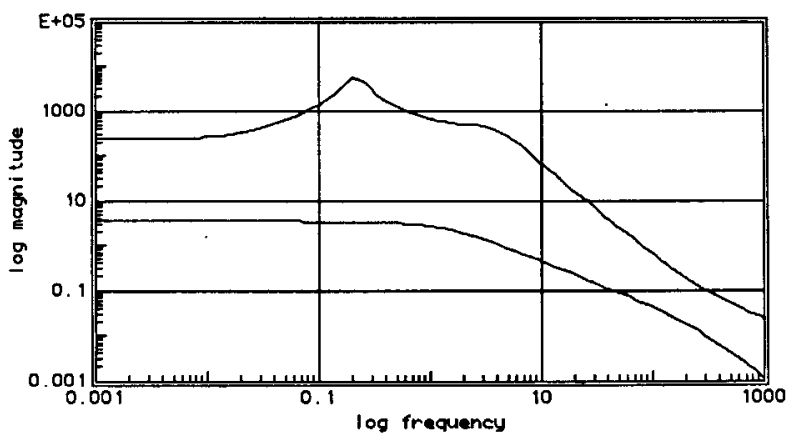

Figure 5. Singular values of the aircraft model.

The performance weighting function for this problem is uniform (see (18)), with

$$
\pi_{\mathrm{p}}(\mathrm{s})=\frac{1}{2}\left(\frac{\mathrm{s}+0.03}{\mathrm{~s}+3}\right)
$$

The uncertainty is modeled as an input multiplicative uncertainty with uniform uncertainty weighting (17):

$$
\pi_{\mathrm{u}}(\mathrm{s})=\frac{1}{2}(0.05 \mathrm{~s}+1)
$$

Bode plots of these weighting functions are shown in Figure 6, and the weighted condition number $\underline{\mathrm{K}} \mathrm{P}(19)$ is shown in Figure 7 .

The design objectives are easily derived from Figure 5 and conditions (27)-(28) and (32)-(34). First, crossover region must be approximately contained in the interval $[2,200]$. Since the weighted condition number is as high as 100 in this region, the singular values must have a separation of approximately 40 $\mathrm{db}$ over the crossover region. If we allow a $20 \mathrm{db} / \mathrm{dec}$ ade rolloff, this separation translates into a 2 decade crossover region, which the same wider as the available region. Thus, we would expect that the robust performance objective will be difficult to achieve. In fact, the compensator presented in [Ste86] using $\mu$-optimization achieves a maximum structured singular value of 1.00 .

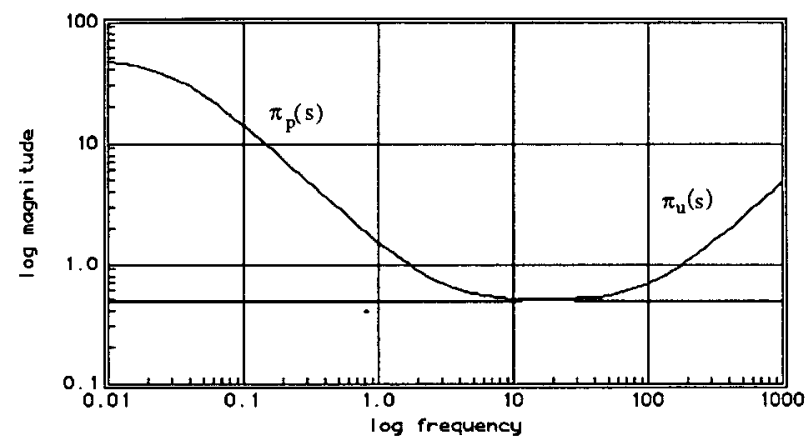

Figure 6. Bode magnitude plots of the weighting functions $\pi_{\mathrm{p}}$ and $\pi_{\mathrm{u}}$.

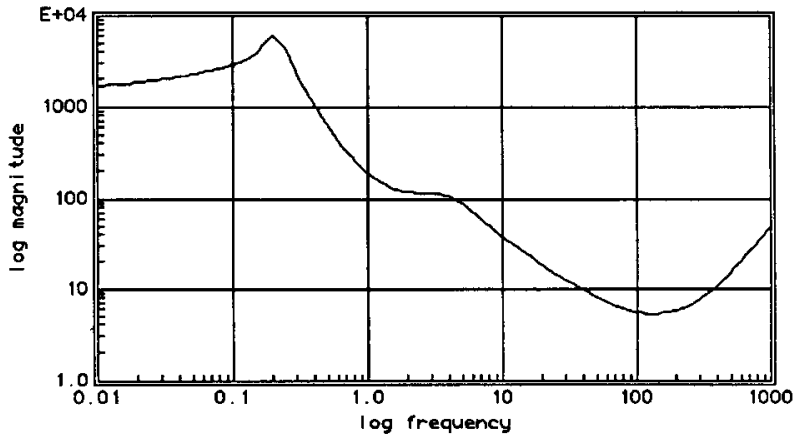

Figure 7. Weighted condition number of the aircraft model.

We will use the input LQG/LTR formal synthesis procedure with the weights selected as described in Section 5. Thus, we select

$$
\begin{aligned}
& \mathrm{W}_{\mathrm{u}}(\mathrm{s})=\frac{1}{2}(0.05 \mathrm{~s}+1) \pi_{l}(\mathrm{~s}) \mathrm{I} \\
& \mathrm{W}_{\mathrm{p}}(\mathrm{s})=\frac{1}{2}\left(\frac{\mathrm{s}+0.03}{\mathrm{~s}+3}\right) \mathrm{W}_{\mu}(\mathrm{s})
\end{aligned}
$$

The high frequency lag $\pi_{l}(\mathrm{~s})$ was selected to cancel the lead in $\mathrm{W}_{\mathbf{u}}(\mathrm{s})$. Thus, $\mathrm{W}_{\mathbf{u}}(\mathrm{s})$ becomes:

$$
\mathrm{W}_{\mathrm{u}}(\mathrm{s})=\frac{1}{2}
$$

The function $W_{\mu}(s)$ was selected initially to be the identity, and the recovery bandwidth was selected to recover the state design up to $350 \mathrm{rad} / \mathrm{sec}$. The singular values of the resulting loop transfer function are shown in Figure 8, and the structured singular value of the resulting closed loop design is shown in Figure 9. Note that the structured singular value has two peaks, one at approximately $7 \mathrm{rad} / \mathrm{sec}$, and one at approximately 150 $\mathrm{rad} / \mathrm{sec}$. The source of these peaks can be traced to two sources. The first factor is that the crossover frequency of the larger loop is slightly too high. The impact of this factor can be reduced by reducing the recovery bandwidth of the design. The second 
factor is that the singular values of the design are further apart than necessary. At the crossover of the first loop, the singular values are separated by more than two decades. This separation is manifested in the double peak that occurs in the structured singular value.

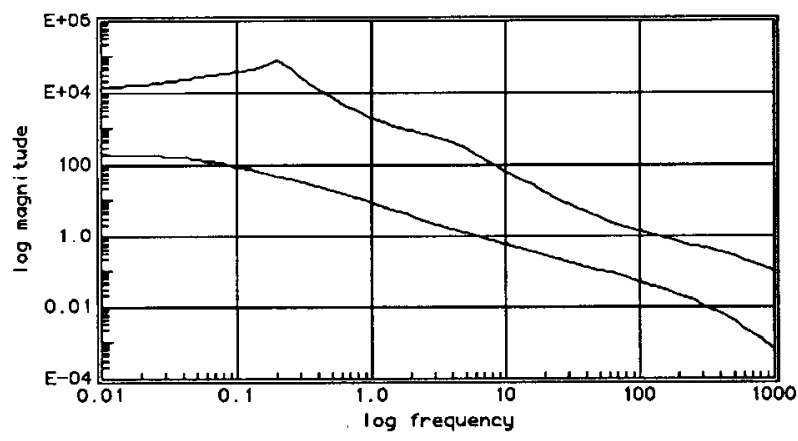

Figure 8. Singular values of the open loop transfer function for the initial design.

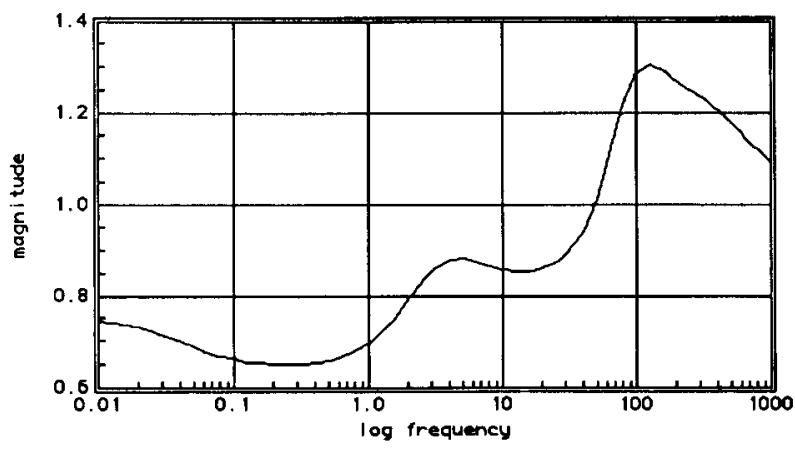

Figure 9. Structured singular value of the initial design.

These observations lead us to modify the design by selecting $\mathrm{W}_{\mu}(\mathrm{s})$ as:

$$
\mathrm{W}_{\mu}(\mathrm{s})=\frac{1}{5} \mathrm{Z}\left[\begin{array}{cc}
0.75 & 0 \\
0 & 1
\end{array}\right] \mathrm{Z}^{\mathrm{H}}
$$

where the matrix $\mathrm{Z}$ is approximately the right singular subspace of the plant transfer function at

$\omega=100 \mathrm{rad} / \mathrm{sec}$. The factor of 0.2 will reduce both singular values by slightly more than 0.5 , while the remainder of $W_{\mu}(s)$ is obtained from (43) to force the singular values closer together.

The singular values of the resulting loop transfer function are shown in Figure 10, and the structured singular value of the resulting closed loop system is shown in Figure 11. Note that the structured singular value now has only one peak (with a shoulder). Also, it should be noted that the maximum of the structured singular value has been reduced to approximately 1.17 .

The structured singular value can be reduced further by noting that we are doing too well at both higher and lower frequencies than is required by the robust performance objective (i.e., the structured singular value is less than unity in these regions). By adding lead to the largest loop singular value above crossover, the structured singular in the crossover region will be reduced (via the mechanism illustrated in (35)).

Similarly, adding lead to the smallest loop singular value below the lowest crossover will reduce the structured singular value in the crossover region. These lead filters can be added through the LQG/LTR procedure. However, the adjustments to the compensator due to these filters will be minor and will not affect the stability of the system. Thus, we can directly place them in series with to the compensator from the second design. Thus our final design $\mathrm{K}_{3}(\mathrm{~s})$ will be constructed by taking the second compensator design $\mathrm{K}_{2}(\mathrm{~s})$ and multiplying it by the lead filter $\mathrm{K}_{l}(\mathbf{s})$ :
$\mathrm{K}_{3}(\mathbf{s})=\mathrm{K}_{l}(\mathbf{s}) \mathrm{K}_{2}(\mathrm{~s})$

The lead filter is selected as:

$$
K_{l}(s)=\left(\frac{s+1.5}{s+2}\right)\left(\frac{0.02 s+1}{0.015 s+1}\right)
$$

The first factor in $K_{l}(s)$ decreases the low frequency gain by a factor of 0.75 below $1.5 \mathrm{rad} / \mathrm{sec}$, while the second factor increases the high frequency gain by a factor of 1.33 above 70 $\mathrm{rad} / \mathrm{sec}$.

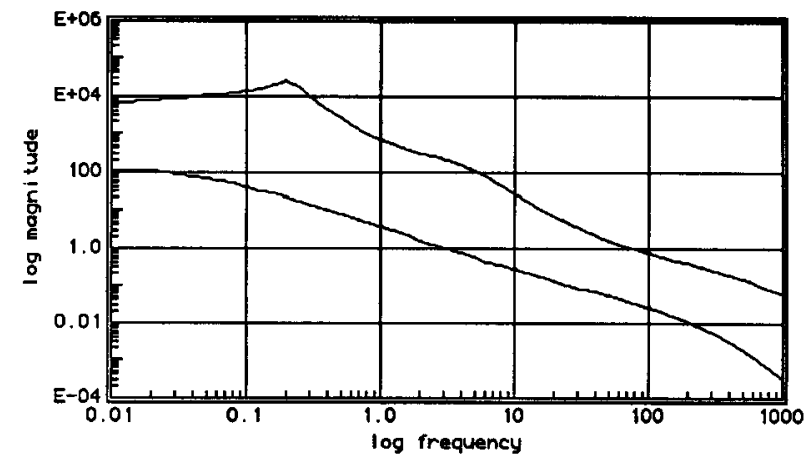

Figure 10. Singular values of the open loop (input) transfer function for the second design.

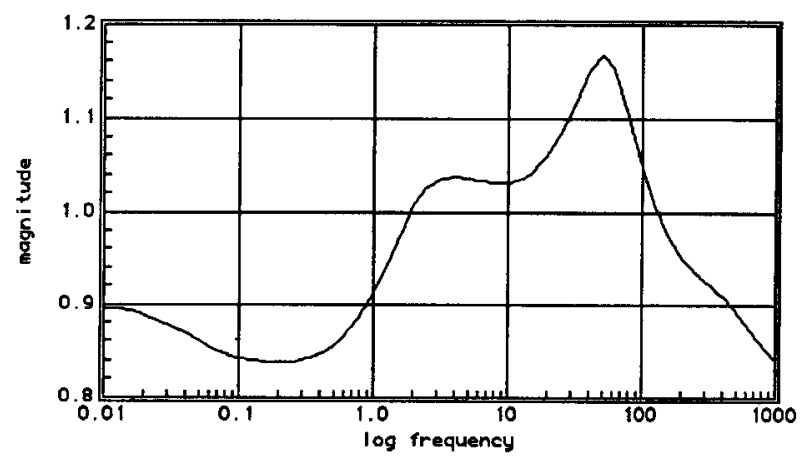

Figure 11. Structured singular value for the second design.

The structured singular value of the resulting design is shown in Figure 12. The peak value of the structured singular value is 1.059 at $30 \mathrm{rad} / \mathrm{sec}$. This value could be reduced further through subsequent applications of the techniques presented in this paper. In particular, additional lead filtering at high frequencies and in the $[0.1,1]$ frequency range coupled with a fine tuning of the open loop singular value separation will lower the structured singular value. However, it should be noted that the structured singular value is within $6 \%$ of the $\mu$-optimal value. The changes required to reduce it further will produce only minor changes in the compensator, and are likely to be dominated by other effects not incorporated in the linearized design problem.

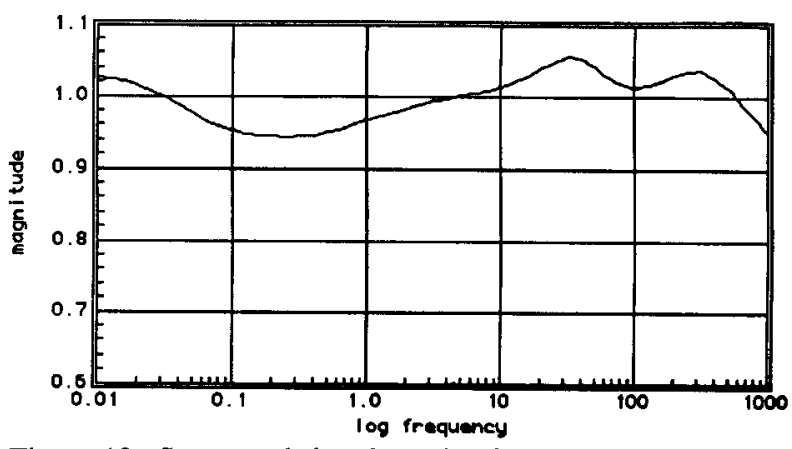

Figure 12. Structured singular value for the final LQ design. 


\section{References}

[Bod45] Bode, H. W., Network Analysis and Feedback Amplifier Design, Van Nostrand, Princeton, NJ, 1945.

[BSM87] Boyd, S. P., G., Stein, and S. K. Mitter, Tutorial Workshop on $H_{\infty}$ Control Theory, Los Angeles, Ca, 1987.

[DoS81] Doyle, J. C., and G. Stein, "Multivariable Feedback Design: Concepts for a Classical/Modern Synthesis," IEEE Trans. A. C., , Vol. AC-26, 1981.

[Doy79] Doyle, J. C., "Robustness of Multiloop Linear Feedback Systems," 17th IEEE CDC, San Diego, CA, 1979.

[Doy82] Doyle, J. C., "Analysis of Feedback Systems with Structured Uncertainties," IEE Proceedings, Vol. 129, Pt. D, No. 6, Nov. 1982.

[Doy83] Doyle, J. C., "Synthesis of Robust Controllers and Filters," 22th IEEE CDC, San Antonio, TX, Dec. 1983.

[Doy84] Doyle, J., "Lecture Notes," 1984 CNR/Honeywell Workshop on Advances in Multivariable Control, Minneapolis, MN, Oct. 1984.

[Doy85] Doyle, J. C., "Structured Uncertainty in Control System Design," 24th IEEE CDC, Fort Lauderdale, FL Dec. 1985.

[DWS82] Doyle, J. C., J. E. Wall, Jr., and G. Stein, "Performance and Robustness Analysis for Structured Uncertainty," 21st IEEE CDC 2 Orlando, FL, Dec. 1982, pp. 629-636.

[FLC82] Freudenberg, J. S., D. P. Looze, and J. B. Cruz, Jr., "Robustness Analysis Using Singular Value Sensitivities," IJC, Vol. 35, 1982.

[Fre88a] Freudenberg, J. S.,"Analysis and Design for IllConditioned Plants" 1988 ACC, Atlanta, GA, 1988.

[Fre88b] Freudenberg, J. S.,"Design of Ill-Conditioned Plants" 27th IEEE CDC, Austin, TX , 1988.

[FrL84] Freudenberg, J. S., and D. P. Looze, "Phase in Multivariable Systems," 23rd IEEE CDC, Las Vegas, NV, 1984.

[FrL86a] Freudenberg, J. S. and D. P. Looze, "The Relation Between Open Loop and Closed Loop Properties of Multivariable Feedback Systems," IEEE Trans. A. C., Vol. AC-31, 1986.

[FrL86b] Freudenberg, J. S., and D. P. Looze, "Relations Between Properties of Multivariable Feedback Systems at Different Loop Breaking Points: Part II," 1986 ACC, Seattle, WA, 1986.

[FrL88] Freudenberg, J. S., and D. P. Looze, Frequency Domain Properties of Scalar and Multivariable Feedback Systems, Springer-Verlag.

[HBG79] Hartmann, G. L., M. F. Barrett, and C. S. Greene, "Control Design for an Unstable Vehicle," Contract Report NAS 4-2578, NASA Dryden Flight Research Center, December 1979.

[LoF88] Looze, D. P., and J. S. Freudenberg, "Linear Quadratic Guassian Design for Robust Performance Objectives," 27th IEEE CDC, Austin, TX, 1988.

[NeM87] Nett, C. N., and V. Manousiouthakis, "Euclidean Condition and Block Relative Gain: Connections, Conjectures and Clarifications," IEEE Trans. A. C., Vol. AC-32, 1987.

[Sta87] Stein, G. and M. Athans, "The LQG/LTR Procedure for Multivariable Feedback Control System Design," IEEE Trans. A. C., Vol. AC-32, No. 2, Feb. 1987, pp. 105-114.

[SkM86] Skogestad, S. and M. Morari, "Control of IllConditioned Plants: High Purity Distillation," AICHE Annual Meeting, 1986.
[SLH81] Safonov, M. G., A. J. Laub, and G. L. Hartmann, "Feedback Properties of Multivariable Systems: The Role and Use of the Return Difference Matrix," IEEE Trans. A. C., Vol. AC-26, 1981.

[ZaF83] Zames, G. and B. A. Francis, "Feedback, Minimax Sensitivity, and Optimal Robustness," IEEE Trans. A. C., Vol AC-28, 1983. 